Artículo

\title{
Concentración y extracción de macronutrimentos en cuatro variedades de fresa
}

\author{
Martin Aguilar Tlatelpa ${ }^{1}$ \\ Víctor Hugo Volke Haller ${ }^{2}$ \\ Prometeo Sánchez García ${ }^{2}$ \\ Mario Pérez Grajales ${ }^{3}$ \\ Marja Liza Fajardo Franco ${ }^{1 \S}$ \\ ${ }^{1}$ Posgrado en Manejo Sustentable de Recursos Naturales-Universidad Intercultural del Estado de Puebla. \\ Calle principal a Lipuntuahuaca s/n, Lipuntahuaca, Huehuetla, Puebla. CP. 73475. (mat7683@gmail.com). \\ ${ }^{2}$ Posgrado en Edafología-Colegio de Postgraduados-Campus Montecillo. Carretera México-Texcoco km \\ 36.5, Montecillo, Texcoco, Estado de México. CP. 56230. (vvolke@colpos.mx; promet@colpos.mx). \\ ${ }^{3}$ Departamento de Fitotecnia-Universidad Autónoma Chapingo. Carretera México-Texcoco km 38.5, \\ Chapingo, Texcoco, Estado de México. CP. 56230. (perezgm7@yahoo.com.mx).
}

${ }^{\S}$ Autor para correspondencia: azilmar@gmail.com.

\section{Resumen}

La nutrición en fresa (Fragaria $x$ ananassa) es un factor fundamental para alcanzar rendimientos y calidad altos del fruto. Para esto deben considerarse las fuentes fertilizantes, la oportunidad y las concentraciones aplicadas. La planta está adaptada a las condiciones subtropicales y templadas, pero es sensible a fluctuaciones iónicas en la solución nutritiva y al contenido de nutrientes en suelo y sustrato. El análisis de la dinámica de absorción de nutrientes es una de las estrategias más usadas para inferir las necesidades en cada etapa fenológica. Para ello surge la necesidad de formular un algoritmo que permita conocer la cantidad de nutriente que la planta requiere en cada etapa fenológica. El objetivo fue determinar, mediante modelos de regresión, la demanda nutrimental durante el ciclo de cultivo de las variedades de fresa Albión, Festival, Jacona y Zamorana. La hipótesis fue que la concentración y absorción de nutrientes es diferencial en cada variedad y etapa fenológica de la planta, además la absorción de nutrientes puede ser descrita mediante modelos de regresión lineal múltiple. El estudio se realizó usando un muestreo completamente aleatorio para la colecta del material vegetal bajo las condiciones de cultivo en campo. La concentración de nutrientes se determinó mediante análisis químico. La extracción nutrimental se obtuvo y se relacionó con cada etapa fenológica. Los valores de referencia para la concentración y extracción nutrimental se obtuvieron para $\mathrm{N}, \mathrm{P}, \mathrm{K}, \mathrm{Ca}, \mathrm{Mg}$ y S, mediante modelos matemáticos que determinan las necesidades nutrimentales de las plantas en cada etapa de su desarrollo.

Palabras clave: Fragaria $x$ ananassa, concentración nutrimental, extracciones de nutrientes, modelos de regresión.

Recibido: junio de 2019

Aceptado: agosto de 2019 


\section{Introducción}

La fresa (Fragaria x ananassa) es una de las frutillas más populares en el mundo. En México ocupa una superficie de 8976 ha y el rendimiento promedio es de $40.3 \mathrm{t} \mathrm{ha}^{-1}$. La producción total anual es de 361928 t, donde los principales estados productores en orden de importancia son: Michoacán, Baja California, Baja California Sur, México, Oaxaca, Morelos y Jalisco (SIAP, 2017). El cultivo genera beneficios económicos para la sociedad mediante la generación de empleos, ya que en cada ciclo de cultivo representa 1158 jornales $\mathrm{ha}^{-1}$ en vivero, plantación comercial e industrialización (Vega, 2007).

Además, se invierte en una gran cantidad de insumos para la agricultura como plásticos de cubierta, sistemas de riego y agroquímicos, beneficiando a las empresas del ramo en las regiones productoras, también introduce divisas por su exportación y fomenta la agricultura por contrato (Lundy, 2007; Vega, 2007). Estos beneficios se logran gracias a la alta tecnificación del cultivo la cual incrementa el rendimiento, la limpieza, calidad y sanidad de la fruta, genera precocidad en la planta, controla malezas, permite el fertirriego, con la finalidad de mejorar la eficiencia del riego y la absorción del fertilizante aplicado. La fertilización es clave para lograr los principales objetivos en la producción de fresa que es incrementar la calidad y cantidad de frutos por unidad de superficie (Jamali et al., 2013). En el valle de Zamora, Michoacán, existen tres sistemas de producción agrupados en función de su nivel tecnológico estos son: el tradicional, intermedio y avanzado. En esta misma región el uso de tecnologías mejoradas inició en 1991 con la introducción del acolchado plástico, cuya ventaja fue controlar las malezas y permitir el fertirriego.

En 1998 se comenzó el uso de túneles con cubierta de plástico para proteger al cultivo de las lluvias tardías y de las bajas temperaturas. Posteriormente, en 2002 se inició el cultivo semi-hidropónico, primero en suelo y posteriormente en sustrato de fibra de coco, procurando disminuir los costos de desinfección de suelo (Vega, 2007). Bajo el contexto anterior la nutrición de la planta constituye un factor fundamental para alcanzar altos rendimientos, en este proceso se deben considerar, las fuentes fertilizantes, la oportunidad y las concentraciones aplicadas.

Cuando esta se hace por fertirriego, ya sea en suelo o sustrato, la cantidad de nutrientes debe considerar: las necesidades de la planta, el estado de desarrollo, la variedad (puede presentar requerimientos nutrimentales diferenciales), el aporte de nutrientes que pueda hacer el suelo o sustrato, la fuente de fertilizante en algunos nutrientes y la eficiencia de aprovechamiento del fertilizante, misma que cambia en suelo o en sustrato (Martínez-Bolaños et al., 2008).

Cuando se usa la estrategia de fertirriego para suplementar nutrimentos a la planta se suelen aplicar soluciones nutritivas ya definidas, en términos de sus relaciones iónicas y concentraciones siendo responsabilidad del técnico o agricultor decidir la oportunidad de aplicación con base en su experiencia; sin embargo, en este tipo de sistemas los productores y técnicos, enfrentan problemas nutricionales de la planta que pueden presentarse como clorosis, amarillamientos y déficit de crecimiento, lo que se refleja en pérdidas de rendimiento (Favela et al., 2006; Petrovic y Yoshida, 2013). Las soluciones nutritivas contienen iones disponibles para la absorción de las plantas, la mayoría poseen mecanismos para absorberlos, sin embargo, la concentración de cada ion tiene efectos diferenciales sobre los procesos fisiológicos, dependiendo en gran medida de la especie y las condiciones ambientales de desarrollo (Tagliavini et al., 2004; Cárdenas-Navarro et al., 2006). 
La planta de fresa está adaptada a las condiciones sub-tropicales y templadas, pero es muy sensible a las fluctuaciones iónicas en solución nutritiva y al contenido de nutrientes tanto en suelo como en sustrato, por lo que, es necesario buscar estrategias que aseguren un suministro óptimo de la nutrición mineral según los requerimientos de la planta y mantener en óptimas condiciones la disponibilidad de nutrientes en el sustrato (Tagliavini et al., 2004; Yadav et al., 2016).

Una de las estrategias más usadas para inferir las necesidades de la planta en cada etapa de su desarrollo es el análisis de la dinámica de absorción de nutrientes, la cual relaciona la diferencia del contenido nutrimental en cada órgano o en planta completa y la acumulación de biomasa entre dos etapas fenológicas, considerando la disponibilidad de cada elemento en el suelo o en el sustrato, para dilucidar las necesidades reales de nutrimento para la planta (Daugaard, 2001; Wójcik y Lewandowski, 2003). Lo anterior, con el objetivo de hacer una dosificación óptima del fertilizante y de esta manera, racionar los recursos y reducir la lixiviación de residuos de fertilizante a horizontes más profundos del suelo, acuíferos, cuerpos de agua o incluso a la atmósfera (Tagliavini et al., 2005).

A este tipo de análisis también se le denomina como curva de absorción de nutrimentos, la cual determina la cantidad de nutrientes que consume una planta; a través, de su ciclo de vida y permite monitorear de forma continua la respuesta de la planta a las condiciones nutrimentales del sustrato, para lograr un balance en las funciones fisiológicas y desarrollo de su máximo potencial genético (Molina et al., 1993; Orona-Castillo et al., 2004; Mattar y Pizarro, 2007).

Una vez identificada la demanda nutrimental, surge la necesidad de formular un algoritmo que permita interpolar la cantidad de nutriente que la planta requiere en cada etapa fenológica, especialmente en los tiempos en que no se han llevado a cabo muestreos. Para tal fin, se crean y diseñan modelos matemáticos. Por tal motivo, el objetivo de esta investigación fue determinar, mediante modelos de regresión, la demanda nutrimental durante el ciclo de cultivo de las variedades de fresa Albión, Festival, Jacona y Zamorana.

\section{Materiales y métodos}

\section{Establecimiento del cultivo}

La presente investigación se llevó a cabo en una parcela con cultivo comercial de fresa en la zona de Zamora, Michoacán, México (19 $9^{\circ}$ 59' 57" latitud norte 102 20' 9"' longitud oeste y altitud de $1570 \mathrm{~m}$ ). El cultivo se desarrolló en un macrotúnel plástico, en sustrato de fibra de coco y con fertirriego, en un sistema altamente tecnificado, donde se evaluaron las variedades Albión, Festival, Jacona y Zamorana. Las plantas se desarrollaron en contenedores de $1 \mathrm{~m}$ x $0.3 \mathrm{~m}$ x $0.1 \mathrm{~m}$ (bolis), donde se colocaron 10 plantas a doble hilera distribuidas en tres bolillos, cada hilera tuvo cinco plantas a una separación de $0.2 \mathrm{~m}$.

El cultivo se fertilizó con la solución nutritiva de Steiner, aplicada en el riego, la cual varió su concentración y conductividad eléctrica según la etapa fenológica del cultivo (Steiner, 1984; Hernández et al., 2006) (Cuadro 1). El control de malezas, plagas y enfermedades se realizó de acuerdo al esquema tecnificado de manejo que realizan los productores de la zona de estudio. El riego se dosificó con el monitoreo constante de la humedad del sustrato. 
Cuadro 1. Composición de la solución nutritiva aplicada en cada etapa fenológica en plantas de fresa cultivadas en sustrato de fibra de coco en Zamora, Michoacán.

\begin{tabular}{|c|c|c|c|c|c|c|c|c|}
\hline \multirow{2}{*}{$\mathrm{DDT}^{\dagger}$} & \multirow{2}{*}{ Etapa fenológica } & \multirow{2}{*}{ 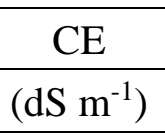 } & $\mathrm{NO}_{3}^{-}$ & $\mathrm{H}_{2} \mathrm{PO}_{4}^{-}$ & $\mathrm{SO}_{4}^{2-}$ & $\mathrm{K}^{+}$ & $\mathrm{Ca}^{2+}$ & $\mathrm{Mg}^{2+}$ \\
\hline & & & \multicolumn{6}{|c|}{$\left(\mathrm{meq} \mathrm{L}^{-1}\right)$} \\
\hline $0-60$ & Vegetativa & 0.5 & 3 & 0.3 & 1.8 & 1.8 & 2.3 & 1 \\
\hline$>60-90$ & Floración & 1 & 6 & 0.5 & 3.5 & 3.5 & 4.5 & 2 \\
\hline$>90-120$ & Cuaje-maduración & 1.3 & 7.5 & 0.6 & 4.4 & 4.4 & 5.6 & 2.5 \\
\hline$>120-165$ & Colecta de fruto & 1.5 & 9 & 0.8 & 5.3 & 5.3 & 6.8 & 3 \\
\hline
\end{tabular}

${ }^{\dagger}=$ días después del trasplante.

\section{Obtención de las muestras}

El material vegetal se obtuvo mediante muestreo completamente aleatorio cada 15 días después del trasplante (DDT) y durante 165 días, con un total de 11 muestreos. En cada muestreo se seleccionaron cuatro plantas por variedad y cada una se consideró como una submuestra.

\section{Variables}

Las variables evaluadas fueron: a) producción de biomasa aérea (formada por hojas, pecíolos, flores, frutos y corona de la planta); y b) concentración y extracción de $\mathrm{N}, \mathrm{P}, \mathrm{K}, \mathrm{Ca}, \mathrm{Mg}$ y $\mathrm{S}$. Paralelamente, se registró la fenología de las plantas de cada variedad (Enz y Dachler, 1998; Woldb y Hutchisona, 2003; Meier et al., 2009). Las plantas se secaron en una estufa con ventilación forzada a $70{ }^{\circ} \mathrm{C}$ por $72 \mathrm{~h}$ y se pesaron para estimar la producción de biomasa. Posteriormente, el tejido seco se molió con un molino tipo Wiley y se almacenó en sobres de papel para continuar con el análisis químico.

La determinación de N se realizó mediante el método Semimicro-Kjeldahl (Alcántar y Sandoval, 1999), mientras que el $\mathrm{P}, \mathrm{K}, \mathrm{Ca}, \mathrm{Mg}$ y S se determinaron mediante una digestión húmeda con una mezcla de ácidos perclórico y nítrico en relación de 2:1 (Alcántar y Sandoval, 1999). La cuantificación del $\mathrm{P}$, Ca y Mg se realizó con un espectrofotómetro de absorción atómica con inducción acoplada a plasma (modelo Liberty Series II, marca Variant Alemania). El K se cuantificó mediante un flamómetro (Corning 400-Flame photometer) y el S mediante un espectrofotómetro (Thermo Genesys 10 series). La extracción nutrimental se estimó a partir del producto de la biomasa aérea y las concentraciones nutrimentales.

\section{Análisis estadístico}

El análisis estadístico de la producción de biomasa aérea más corona, concentración y extracción nutrimental se realizó mediante análisis de regresión considerando la etapa fenológica, según el método descrito por Volke (2008), donde se especifica un modelo con unas pocas variables a partir de las relaciones gráficas entre las variables respuesta y los factores de estudio, donde se anexan variables al modelo con base en la relación gráfica entre los residuos y los factores aun no incluidos en el modelo, que mostraron alguna tendencia de respuesta, hasta obtener el modelo con el menor cuadrado medio del error (CME) y mayor coeficiente de determinación $\left(\mathrm{R}^{2}\right)$ (Montoya-García et al., 2018). 
Para cada variedad y nutrimento se generó un modelo de regresión en relación con la etapa fenológica del ciclo de cultivo. Los modelos de regresión se obtuvieron con SAS ${ }^{\circledR}$ versión 9.0 y las gráficas de la concentración nutrimental se generaron con los valores estimados por los modelos.

\section{Resultados y discusión}

\section{Etapas fenológicas del cultivo}

Las etapas fenológicas que se identificaron durante el estudio y se relacionaron con la concentración nutrimental fueron: 1) 4 a 5 hojas (15 DDT); 2) aparición de flores primarias (30 DDT); 3) emisión de estolones (45 DDT); 4) incremento de follaje (60 DDT); 5) floración (75 DDT); 6) frutos blancos (90 DDT); 7) desarrollo de frutos (105 DDT); y 8) fructificación (120 a 165 DDT). El desarrollo fenológico de las variedades Jacona y Zamorana ocurrió con un retraso de 15 días en relación con las variedades Albión y Festival, lo que se tradujo en un ciclo de desarrollo 15 días más largo para las primeras. Debido a lo anterior, Albión y Festival fructificaron de manera temprana en comparación con Jacona y Zamorana.

\section{Producción de biomasa aérea}

Los modelos de regresión que describieron la dinámica en la acumulación de materia seca en las variedades de fresa estudiadas fueron los siguientes:

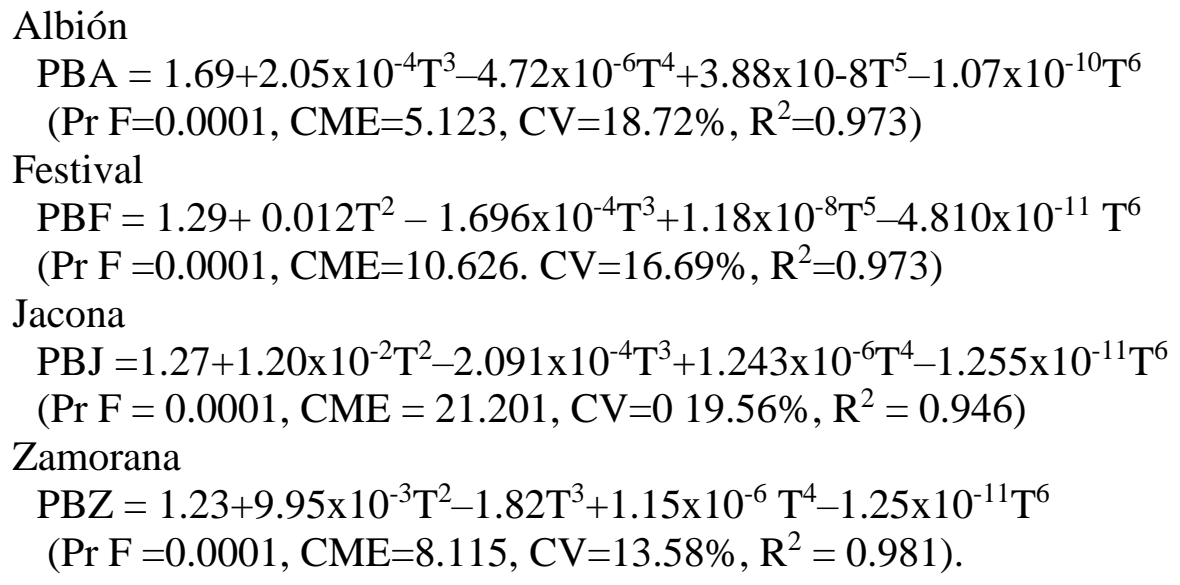

Donde: $\mathrm{PB}=$ producción de biomasa aérea más coronas $\left(\mathrm{g}_{\mathrm{planta}} \mathrm{-}^{-1}\right.$, base seca), en cada variedad de fresa; $\mathrm{T}=$ días a partir del trasplante hasta 165 días; $\mathrm{Pr} \mathrm{F}=$ probabilidad de $\mathrm{F}$; $\mathrm{CME}=$ cuadrado medio del error; $\mathrm{CV}=$ coeficiente de variación; $\mathrm{R}^{2}=$ coeficiente de determinación múltiple.

Estos modelos tuvieron el mejor ajuste para la producción de biomasa acumulada en cada variedad durante el ciclo de cultivo ( $\operatorname{Pr} \mathrm{F} \leq 0.0001, \mathrm{R}^{2}>0.9$ ). Albión presentó menor producción de biomasa aérea en comparación con Festival, Jacona y Zamorana, esta respuesta puede deberse a que Albión se caracteriza por tener un desarrollo lento en comparación con otras variedades, presenta menor porte y es susceptible a plagas como la araña roja, aunque tiene una elevada plasticidad fenotípica (Shaw y Larson, 2005; Costa et al., 2017; Esteca et al., 2017). 
La producción de biomasa presentó tres etapas de mayor acumulación: la primera, de los 15 a los 30 DDT, donde se observó un rápido desarrollo del área foliar; la segunda de los 90 a los 120 DDT, que se caracterizó por un rápido desarrollo vegetativo y la tercera de los 150 a los 165 DDT, donde se alcanzó el máximo desarrollo, mostrando síntomas de envejecimiento y pérdida de hojas.

Los mayores incrementos en biomasa se presentaron de los 90 a los 135 DDT en todas las variedades, debido principalmente al desarrollo de los órganos reproductivos (Figura 1). Lo anterior coincidió con los resultados de Molina et al. (1993); Tagliavini et al. (2005) quienes observaron que el crecimiento de la variedad Chandler, fue muy bajo durante las primeras nueve semanas (63 días); mientras que entre los días 113 a 168 se presenta la mayor producción de biomasa, misma que coincide con el pico de fructificación.

Después de 155 días bajó la acumulación de biomasa por la defoliación y la demanda de energía que la fructificación requiere. Este comportamiento se presenta en cada evento de fructificación, donde el fruto es de menor calidad en cada fructificación sucesiva (Avitia-García et al., 2014) (Figura 1).

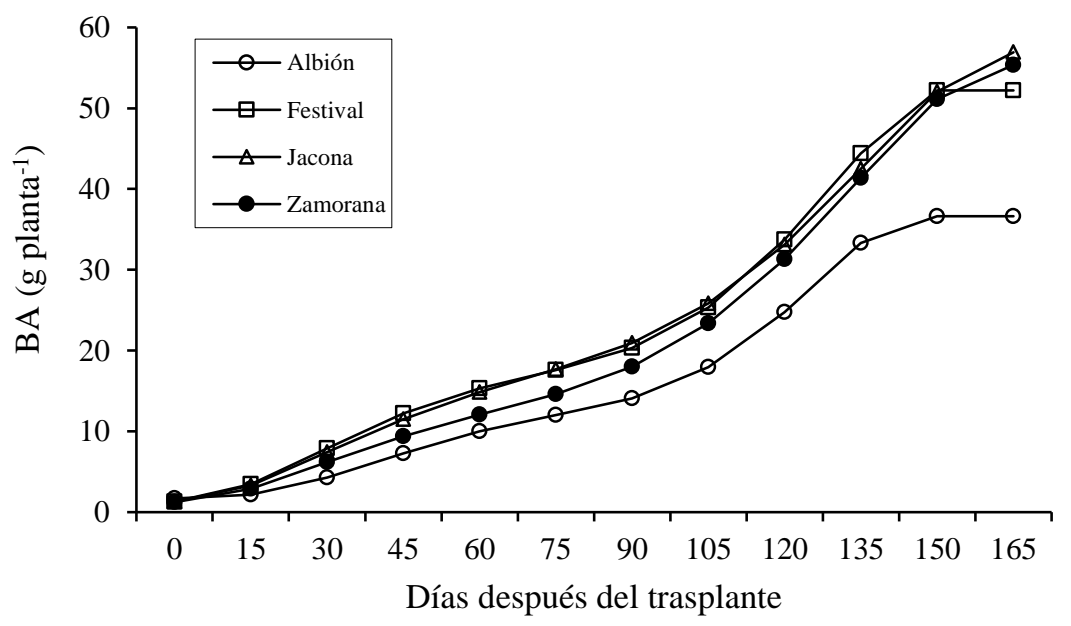

Figura 1. Producción acumulada de biomasa aérea para cuatro variedades de fresa cultivadas en fibra de coco con un sistema hidropónico estimadas mediante modelos de regresión. $\mathrm{BA}=$ biomasa aérea acumulada.

\section{Concentración nutrimental en parte aérea más corona}

Los modelos de regresión con mejor ajuste para cuantificar la concentración nutrimental en cada una de las variedades de fresa en función de su etapa fenológica fueron los siguientes:

Nitrógeno

$\mathrm{CN}=1.72-0.16 \mathrm{~V}_{2}-0.29 \mathrm{~V}_{3}-0.179 \mathrm{~V}_{4}-7.75 \times 10^{-3} \mathrm{~T}+1.041748 \times 10^{-5} \mathrm{~T}^{3}-1.9 \times 10^{-7} \mathrm{~T}^{4}+1.23 \times 10^{-9} \mathrm{~T}^{5}-$

$2.73 \times 10^{-12} \mathrm{~T}^{6}$

$\left(\mathrm{Pr} F=0.0001, \mathrm{CME}=0.0046, \mathrm{CV}=4.158 \%, \mathrm{R}^{2}=0.856\right)$

Fósforo

Albión, Festival, Zamorana

$\mathrm{CP}=0.41+2.39 \times 10^{-6} \mathrm{~T}^{3}-4.56 \times 10^{-8} \mathrm{~T}^{4}+3.2 \times 10^{-10} \mathrm{~T}^{5}-7.82 \times 10^{-13} \mathrm{~T}^{6}+0.37 \mathrm{P}_{15}$

$\left(\mathrm{Pr} F=0.0001, \mathrm{CME}=0.00103, \mathrm{CV}=5.25 \%, \mathrm{R}^{2}=0.948\right)$ 


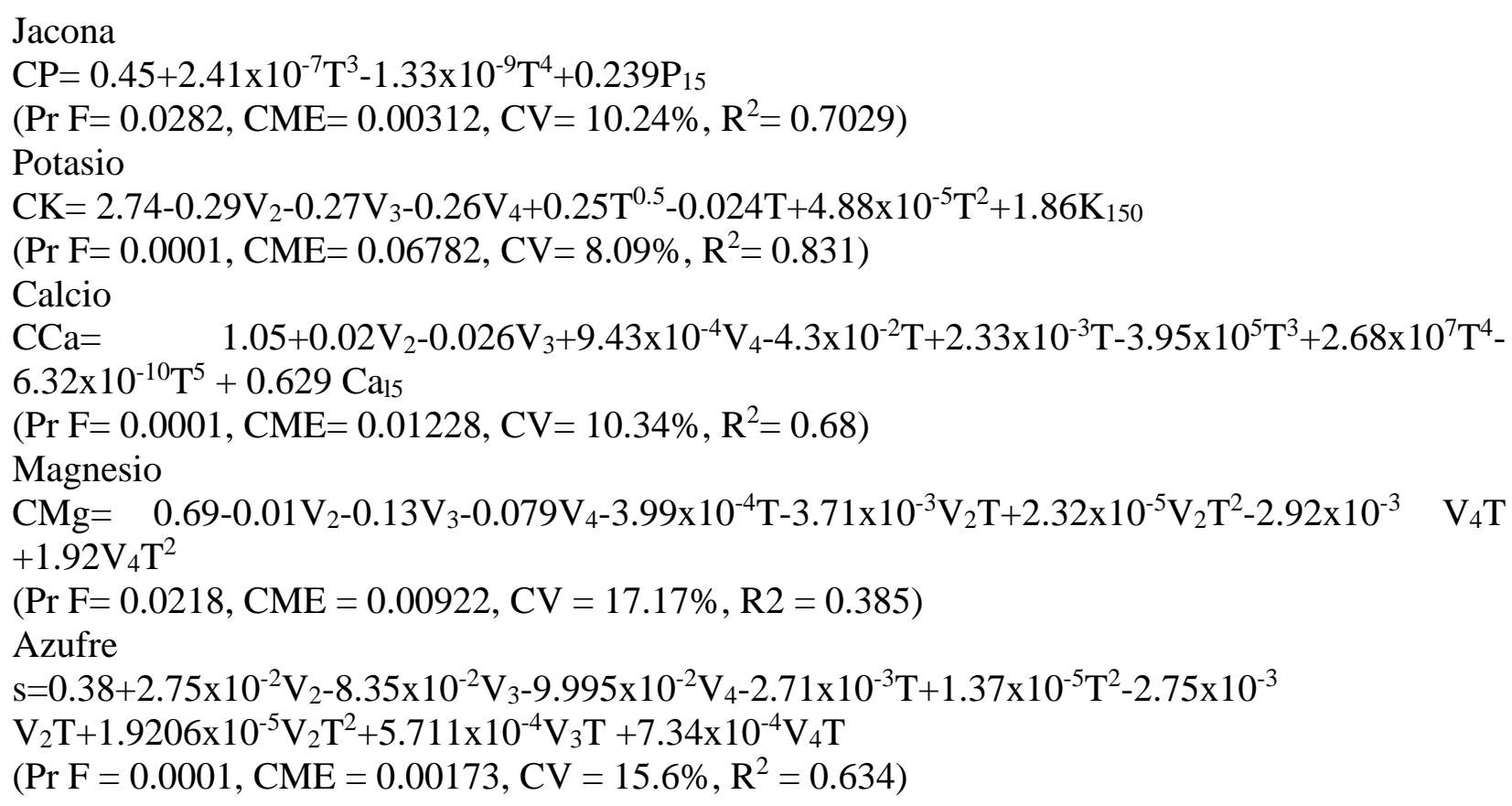

Donde: $\mathrm{CN}, \mathrm{CP}, \mathrm{CK}, \mathrm{CCa}, \mathrm{CMg}$ y $\mathrm{Cs}$ son las concentraciones de $\mathrm{N}, \mathrm{P}, \mathrm{K}, \mathrm{Ca}, \mathrm{Mg}$ y S (porcentaje en base seca); $V_{2}, V_{3}$ y $V_{4}$ son variables auxiliares para las variedades Festival, Jacona y Zamorana; $\mathrm{T}=$ días a partir del trasplante hasta $165 ; \mathrm{P}_{15}, \mathrm{~K}_{150}$ y $\mathrm{Ca}_{15}$ son variables auxiliares para los periodos de 15 y 150 días en que las variedades presentaron concentraciones más altas que la tendencia observada. $\operatorname{Pr} \mathrm{F}=$ probabilidad de $\mathrm{F}, \mathrm{CME}=$ cuadrado medio del error, $\mathrm{CV}=$ coeficiente de variación, $\mathrm{R}^{2}=$ coeficiente de determinación múltiple. Todos los coeficientes de regresión fueron significativos $(\operatorname{Pr} \mathrm{F} \leq 0.01)$

Los valores de concentración nutrimental en la biomasa aérea más corona estimados con los modelos se presentan en las Figuras 2 y 3. En la Figura 2A, se observa la concentración nutrimental de $\mathrm{N}$ en las cuatro variedades estudiadas, las concentraciones se posicionaron en el intervalo de 1.3 a $1.95 \%$, bajas en comparación de lo reportado por Cárdenas-Navarro et al. (2006), donde la concentración varió de 1.5 a $2.9 \%$, en un ensayo de diversas relaciones amonio/nitrato.

Sin embargo, Castro et al. (2005) aplicaron concentraciones marginales de $\mathrm{N}$ donde encontraron que las plantas expresaron deficiencia de nitrógeno a la concentración de $1.1 \%$ en tejido foliar, al final del ciclo de cultivo. Molina et al. (1993), encontraron que a lo largo del ciclo de 30 semanas (210 días) la concentración fue de 1.4 a $2.86 \%$, las variaciones en el extremo superior pueden deberse a la dosificación de $\mathrm{N}$ en cada experimento en particular. El nitrógeno debe usarse con cuidado para no disminuir la calidad del fruto, la firmeza, el contenido de ácido ascórbico e incrementando el contenido de polifenoles (Castro et al., 2005; Agulheiro-Santos, 2009).

En la Figura 2B, se presentan las concentraciones de fósforo $(\mathrm{P})$ para las cuatro variedades de fresa, que oscilaron entre 0.41 a $0.78 \%$, valores que coinciden con las encontradas en las variedades Aromas, Seascape, Chandler, Diamante y Pajaro, que presentaron deficiencias de este nutrimento a concentraciónes $>0.15 \%$ (Muramoto, 2003). Sin embargo, la variedad Chandler presentó concentraciones mas bajas durante su ciclo estas fluctuarón de entre 0.16 a $0.44 \%$ (Molina et al., 1993), aunque se han reportado concentraciones tan bajas como de 0.19 a 0.35\% (Daugaard, 2001). 
Las etapas fenológicas donde la planta incrementó la concentración de P fue en el desarrollo del fruto y la fructificación, lo que indica la importancia de asegurar la disponibilidad de fósforo en estas etapas críticas, dado que las plantas deficientes pueden desarrollar flores y frutos pequeños en comparación con plantas no deficientes (Nestby et al., 2004).

Las concentraciones de potasio (K) oscilaron entre 2.46 a $3.42 \%$ en las cuatro variedades, lo cual es mayor a lo reportado por Molina et al. (1993), en la variedad Chandler que tuvo concentraciones entre $1.33 \%$ a 2.52\%. Mientras que Daugaard (2001), encontró concentraciones de entre $1.5 \%$ a $1.8 \%$ durante todo el ciclo del cultivo en un promedio de ocho variedades de fresa (Figura 2C). En la presente investigación, las diferencias de concentración de K entre las etapas iniciales y finales fueron del orden del $1 \%$ en cada variedad. Las etapas fenológicas donde se observó la mayor concentración de este nutrimento coinciden con la aparición de las primeras flores en los días 30 y 45, aunque, las concentraciones de $\mathrm{K}$ en el tejido vegetal constituyeron del 1 al $5 \%$ de la materia seca, lo cual se considera suficiente al ser mayor a $1.5 \%$ (Favela et al., 2006; Mattar y Pizarro, 2007).
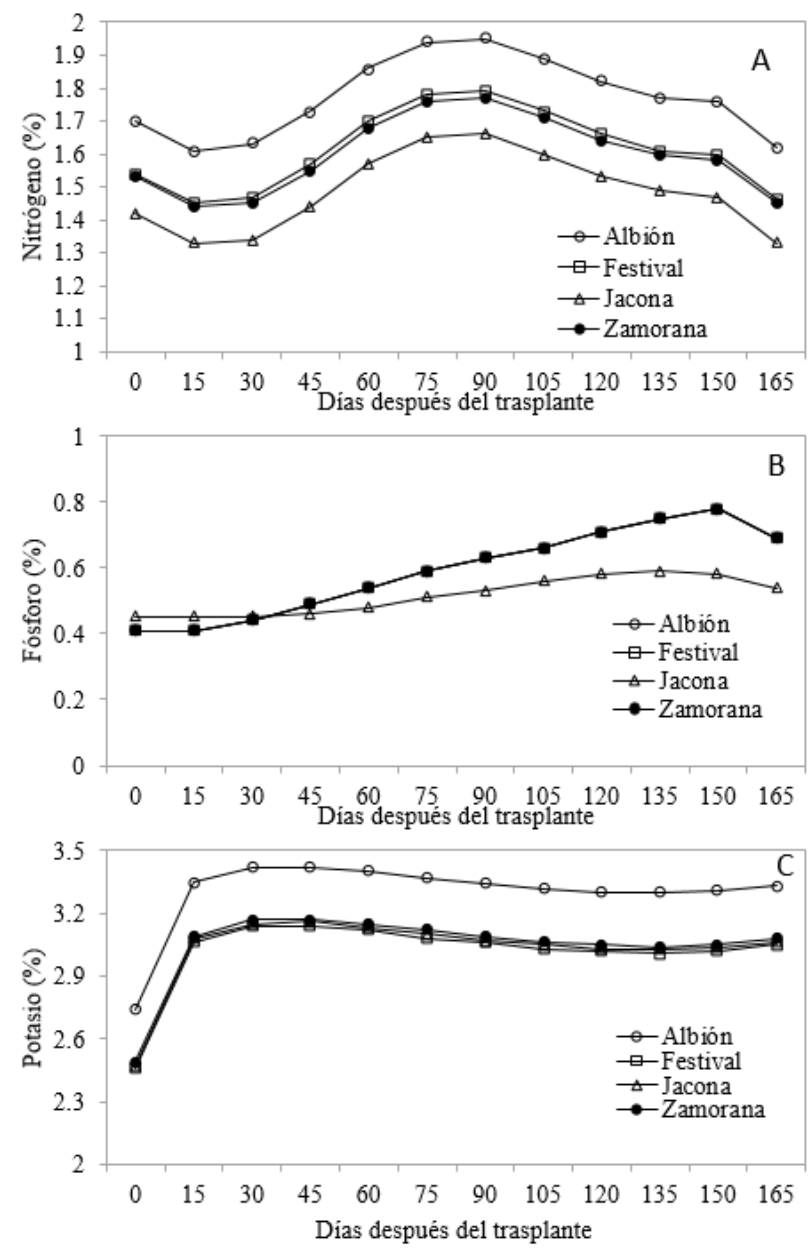

Figura 2. Concentración de macronutrimentos en biomasa aérea más corona en función del tiempo para cuatro variedades de fresa. A) nitrógeno; B) fósforo; y C) potasio. 
La concentración de calcio (Ca) osciló entre 0.76 a 1.27\% (Figura 3A), coincidiendo con los valores reportados por Molina et al. (1993), donde las concentraciones oscilaron entre 0.47 a $1.9 \%$ y de Daugaard (2001), cuyas concentraciones oscilaron entre 0.9 y $1.9 \%$; la concentración óptima puede situarse entre 0.7 y $1.2 \%$ (Nestby et al., 2004) y los síntomas de deficiencia se pueden observar a concentraciones por debajo de 0.5\% (Mattar y Pizarro, 2007).

Las concentraciones de magnesio $(\mathrm{Mg})$ fueron homogéneas a lo largo del ciclo de cultivo dado que oscilaron de entre 0.46 a $0.68 \%$, niveles suficientes para la planta (Favela et al., 2006), las concentraciones mayores se presentaron al inicio y final del ciclo de cultivo; en otros estudios (Daugaard, 2001; Molina et al., 1993), se han encontrado concentraciones que oscilan entre 0.19 a $0.65 \%$, cuyos valores coinciden con los encontrados en este trabajo (Figura 3B). Las concentraciones de azufre oscilaron entre 0.18 a $0.41 \%$, la concentración de este elemento en el tejido vegetal fue constante a lo largo del ciclo de cultivo (Figura 3C). Los síntomas de deficiencia se presentan solo a concentraciones inferiores de $0.01 \%$, las concentraciones óptimas en hoja oscilan entre 0.15 y $0.5 \%$ (Olivia et al., 2017).
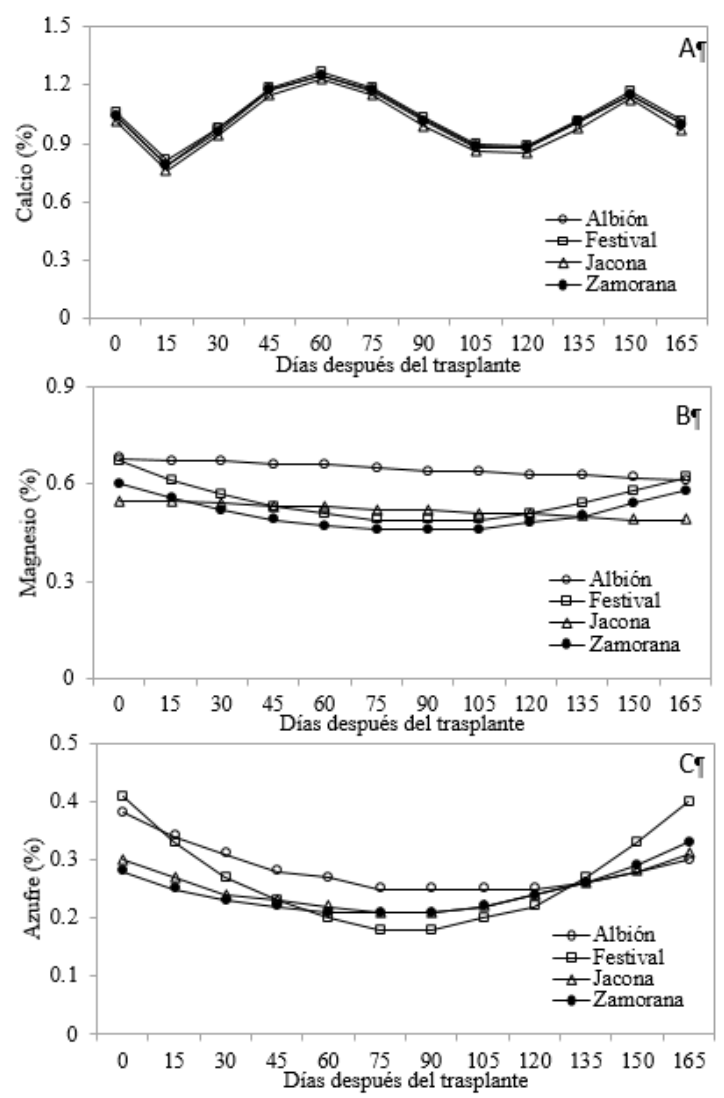

Figura 3. Concentración de macronutrimentos en biomasa aérea más corona en función del tiempo para cuatro variedades de fresa. A) calcio; B) magnesio; y C) azufre.

Las concentraciones de todos los elementos en las cuatro variedades estuvieron dentro de los límites permisibles por lo que no se observaron síntoma de deficiencia en ninguna de las variedades evaluadas. Por tal motivo, los valores promedio de la concentración nutrimental de las cuatro variedades pueden ser utilizados como referentes, siempre y cuando la metodología de cuantificación sea homóloga. 


\section{Extracción nutrimental}

Para cada variedad de fresa se desarrollaron seis modelos, uno para cada nutriente analizado (N, $\mathrm{P}$, $\mathrm{K}, \mathrm{Ca}, \mathrm{Mg}, \mathrm{S}$ ), resultando 24 modelos que predicen la extracción de los elementos en función del tiempo. Estos últimos se pueden relacionar con la etapa fenológica del cultivo, únicamente al sustituir los parámetros para obtener la extracción de nutrientes expresada en kilogramos por hectárea. Los modelos para estimar la extracción nutrimental en base al producto de la concentración de cada elemento y el contenido de biomasa aérea en $\mathrm{N}, \mathrm{P}, \mathrm{K}, \mathrm{Ca}, \mathrm{Mg}$ y S en cada variedad de fresa se expresaron en unidades de masa (g) en 50000 plantas de fresa dado que esta es una densidad de población promedio en las zonas de cultivo, sin embargo, cada ecuación se puede ajustar según la densidad de población deseada (Cuadro 2 y 3).

\section{Cuadro 2. Ecuaciones de extracción nutrimental para las variedades Albión y Festival.}

\begin{tabular}{|c|c|c|c|c|c|}
\hline $\mathrm{Ex}^{\dagger}$ & Modelo $^{\text {II }}$ & CME & $\mathrm{CV}$ & $\mathrm{R}^{2}$ & $\operatorname{Pr} \mathrm{F}$ \\
\hline \multicolumn{6}{|c|}{ Albión } \\
\hline $\mathrm{N}$ & $1.8 \times 10^{-1} \mathrm{~T}^{3}-5.7 \times 10^{-3} \mathrm{~T}^{4}+6.6 \times 10^{-5} \mathrm{~T}^{5}-3.2 \times 10^{-7} \mathrm{~T}^{6}+5.5 \times 10^{-10} \mathrm{~T}^{7}$ & 2413.6 & 1.6 & 0.9 & $* *$ \\
\hline $\mathrm{P}$ & $3.4 \times 10^{-1} \mathrm{~T}^{2}+2.7 \times 10^{-2} \mathrm{~T}^{3}-9.1 \times 10^{-4} \mathrm{~T}^{4}+8.9 \times 10^{-6} \mathrm{~T}^{5}-2.8 \times 10^{-8} \mathrm{~T}^{6}$ & 175.8 & 1 & 0.9 & $* *$ \\
\hline $\mathrm{K}$ & $6.5 \mathrm{~T}^{2}-4.3 \times 10^{-3} \mathrm{~T}^{4}+6.6 \times 10^{-5} \mathrm{~T}^{5}-3.6 \times 10^{-7} \mathrm{~T}^{6}+6.4 \times 10^{-10} \mathrm{~T}^{7}$ & 5112.3 & 1.2 & 0.9 & $* *$ \\
\hline $\mathrm{Ca}$ & $1.4 \times 10^{-1} \mathrm{~T}^{3}-4.2 \times 10^{-3} \mathrm{~T}^{4}+4.6 \times 10^{-5} \mathrm{~T}^{5}-2.1 \times 10^{-7} \mathrm{~T}^{6}+2.9 \times 10^{-10} \mathrm{~T}^{7}$ & 37839 & 9.6 & 0.9 & $* *$ \\
\hline $\mathrm{Mg}$ & $7.6 \times 10^{-1} \mathrm{~T}^{2}+2.8 \times 10^{-2} \mathrm{~T}^{3}-1.4 \times 10^{-3} \mathrm{~T}^{4}+1.9 \times 10^{-5} \mathrm{~T}^{5}-1.1 \times 10^{-7} \mathrm{~T} 6+1.9 \times 10^{-10} \mathrm{~T}^{7}$ & 1204 & 3.2 & 0.9 & $* *$ \\
\hline S & $4.9 \times 10^{-1} \mathrm{~T}^{2}-3.1 \times 10^{-4} \mathrm{~T}^{4}+5.1 \times 10^{-6} \mathrm{~T}^{5}-2.8 \times 10^{-8}+4.9 \times 10^{-11} \mathrm{~T}^{7}$ & 687.3 & 5.4 & 0.9 & $* *$ \\
\hline \multicolumn{6}{|c|}{ Festival } \\
\hline $\mathrm{N}$ & $41.9 \mathrm{~T}+6.7 \mathrm{~T}^{2}-0.2 \mathrm{~T}^{3}+1.9 \times 10^{-3} \mathrm{~T}^{4}-5.9 \times 10^{-6} \mathrm{~T}^{5}$ & 1513.9 & 1 & 0.9 & $* *$ \\
\hline $\mathrm{P}$ & $3.3 \mathrm{~T}^{2}-0.10 \mathrm{~T}^{3}+1.5 \times 10^{-3} \mathrm{~T}^{4}-1.2 \times 10^{-5} \mathrm{~T}^{5}+5.7 \times 10^{-8} \mathrm{~T}^{6}$ & 79.1 & 0.4 & 0.9 & $* *$ \\
\hline $\mathrm{K}$ & $45.7 \mathrm{~T}+25.8 \mathrm{~T}^{2}-1.03 \mathrm{~T}^{3}+1.6 \times 10^{-2} \mathrm{~T}^{4}-1.2 \times 10^{-4} \mathrm{~T}^{5}+4.8 \times 10^{-7} \mathrm{~T}^{6}-7.9 \times 10^{-10} \mathrm{~T}^{7}$ & 3517.4 & 0.8 & 0.9 & $* *$ \\
\hline & $4.3 \mathrm{~T}^{2}-2.9 \times 10^{-3} \mathrm{~T}^{4}+4.4 \times 10^{-5} \mathrm{~T}^{5}-2.33 \times 10^{-7} \mathrm{~T}^{6}+4.1 \times 10^{-10} \mathrm{~T}^{7}$ & 40823 & 6.8 & 0.9 & $* *$ \\
\hline $\mathrm{Mg}$ & $5.7 \mathrm{~T}^{2}-0.24 \mathrm{~T}^{3}+4.1 \times 10^{-3} \mathrm{~T}^{4}-3.5 \times 10^{-5} \mathrm{~T}^{5}+1.6 \times 10^{-7} \mathrm{~T}^{6}-2.8 \times 10^{-10} \mathrm{~T}^{7}$ & 416 & 1.4 & 0.9 & $* *$ \\
\hline
\end{tabular}

$\dagger=$ extracción nutrimental en $\mathrm{g}$ en 50000 plantas; ${ }^{q} \mathrm{~T}=$ días a partir del trasplante. Todos los coeficientes de regresión fueron significativos $(\operatorname{Pr} F \leq 0.01)$.

Estos modelos de regresión permitieron estimar los requerimientos de nutriente para la planta en función de la etapa fenológica en cada variedad. Sin embargo, para su implementación se debe tomar en cuenta que cada modelo no considera la eficiencia del uso del fertilizante ya que esta depende del sistema de cultivo empleado (suelo, goteo, hidroponía, sustrato).

Por lo tanto, las dosificaciones deberán ajustarse según la eficiencia agronómica o de recuperación del fertilizante de acuerdo con lo sugerido por Stewart (2007). Este mismo autor menciona, que la eficiencia de recuperación de los nutrientes son las siguientes: $\mathrm{N}$ de 50 a $70 \%, \mathrm{P}$ de 10 a $25 \%, \mathrm{~K}$ de 50 a $60 \%$, estos dos últimos tienen potencial de acumulación en el suelo, por lo que también se debe considerar este factor al momento de establecer un programa de fertilización. 
Cuadro 3. Ecuaciones de extracción nutrimental para las variedades Jacona y Zamorana.

\begin{tabular}{|c|c|c|c|c|c|}
\hline $\mathrm{Ex}^{\dagger}$ & Modelo ${ }^{\text {TI }}$ & $\mathrm{CME}$ & $\mathrm{CV}$ & $\mathrm{R}^{2}$ & $\operatorname{Pr} \mathrm{F}$ \\
\hline \multicolumn{6}{|c|}{ Jacona } \\
\hline $\mathrm{N}$ & $56.5 \mathrm{~T}+3.6 \mathrm{~T}^{2}-0.11 \mathrm{~T}^{3}+1.01 \times 10^{-3} \mathrm{~T} 4-2.9 \times 10^{-7} \mathrm{~T} 5$ & 1800.6 & 1.1 & 0.9 & ** \\
\hline $\mathrm{P}$ & $21.5 \mathrm{~T}+1.4 \mathrm{~T}^{2}-5.6 \times 10^{-2} \mathrm{~T}^{3}+6.8 \times 10^{-4} \mathrm{~T}^{4}-3.14 \times 10^{-6} \mathrm{~T}^{5}+4.5 \times 10^{-9} \mathrm{~T}^{6}$ & 733.2 & 2 & 0.9 & $* *$ \\
\hline $\mathrm{K}$ & $106.9 \mathrm{~T}+17.9 \mathrm{~T}^{2}-0.8 \mathrm{~T}^{3}+1.2 \times 10^{-2} \mathrm{~T} 4-8.9 \times 10^{-5} \mathrm{~T}^{5}+3.4 \times 10^{-7} \mathrm{~T}^{6}-5.3 \times 10^{-10} \mathrm{~T}^{7}$ & 8782 & 1.2 & 0.9 & $* *$ \\
\hline $\mathrm{Ca}$ & $3.8 \mathrm{~T}^{2}-2.4 \times 10^{-3} \mathrm{~T} 4+3.5 \times 10^{-5} \mathrm{~T}^{5}-1.8 \times 10^{-7} \mathrm{~T}^{6}+3.1 \times 10^{-10} \mathrm{~T}^{7}$ & 19289 & 4.8 & 0.9 & $* *$ \\
\hline $\mathrm{Mg}$ & $10.5 \mathrm{~T}+3.6 \mathrm{~T}^{2}-0.15 \mathrm{~T}^{3}+2.2 \times 10^{-3} \mathrm{~T}^{4}-1.69 \times 10^{-5} \mathrm{~T}^{5}+6.4 \times 10^{-8} \mathrm{~T}^{6}-9.9 \times 10^{-11} \mathrm{~T}^{7}$ & 519.6 & 1.8 & 0.9 & $* *$ \\
\hline $\mathrm{S}$ & $7.8 \mathrm{~T}+1.5 \mathrm{~T}^{2}-6.7 \times 10^{-2} \mathrm{~T}^{3}+1.2 \times 10^{-3} \mathrm{~T}^{4}-9.6 \times 10^{-6} \mathrm{~T}^{5}+4.05 \times 10^{-8} \mathrm{~T}^{6}-7.01 \times 10^{-11} \mathrm{~T}^{7}$ & 199.3 & 1.7 & 0.9 & $*$ \\
\hline \multicolumn{6}{|c|}{ Zamorana } \\
\hline $\mathrm{N}$ & $21.9 \mathrm{~T}+6.7 \mathrm{~T}^{2}-0.3 \mathrm{~T}^{3}+3.9 \times 10^{-3} \mathrm{~T}^{4}-3.2 \times 10^{-5} \mathrm{~T}^{5}+1.4 \times 10^{-7} \mathrm{~T}^{6}-2.6 \times 10^{-10} \mathrm{~T}^{7}$ & 979 & 0.8 & 0.9 & $* *$ \\
\hline $\mathrm{P}$ & $2.8 \mathrm{~T}^{2}-0.10 \mathrm{~T}^{3}+1.6 \times 10^{-3} \mathrm{~T}^{4}-1.4 \times 10^{-5} \mathrm{~T}^{5}+7.04 \times 10^{-8} \mathrm{~T}^{6}-1.5 \times 10^{-10} \mathrm{~T}^{7}$ & 39.6 & 0.3 & 0.9 & $* *$ \\
\hline $\mathrm{K}$ & $105.1 \mathrm{~T}+13.5 \mathrm{~T}^{2}-0.61 \mathrm{~T}^{3}+9.7 \times 10^{-3} \mathrm{~T}^{4}-7.3 \times 10^{-5} \mathrm{~T}^{5}+2.8 \times 10^{-7} \mathrm{~T}^{6}-4.3 \times 10^{-10} \mathrm{~T}^{7}$ & 5516.7 & 1 & 0.9 & $* *$ \\
\hline $\mathrm{Ca}$ & $4.21 \mathrm{~T}^{2}-7.8 \times 10^{-2} \mathrm{~T}^{3}+6.12 \times 10^{-6} \mathrm{~T}^{5}-2.6 \times 10^{-8} \mathrm{~T}^{6}$ & 16645 & 4.5 & 0.9 & $* *$ \\
\hline $\mathrm{Mg}$ & $12.4 \mathrm{~T}+2.6 \mathrm{~T}^{2}-0.12 \mathrm{~T}^{3}+1.96 \times 10^{-3} \mathrm{~T}^{4}-1.6 \times 10^{-5} \mathrm{~T}^{5}+6.6 \times 10^{-8} \mathrm{~T}^{6}-1.13 \times 10^{-10} \mathrm{~T}^{7}$ & 540.1 & 1.6 & 0.9 & $* *$ \\
\hline $\mathrm{S}$ & $6.2 \mathrm{~T}+1.1 \mathrm{~T}^{2}-5.1 \times 10^{-2} \mathrm{~T}^{3}+8.9 \times 10^{-4} \mathrm{~T}^{4}-7.5 \times 10^{-6} \mathrm{~T}^{5}+3.24 \times 10^{-8} \mathrm{~T}^{6}-5.8 \times 10^{-11} \mathrm{~T}^{7}$ & 125.2 & 1.4 & 0.9 & * \\
\hline
\end{tabular}

$\dagger=$ extracción nutrimental en g en 50000 plantas; ${ }^{\mathrm{I}} \mathrm{T}=$ días a partir del trasplante. Todos los coeficientes de regresión fueron significativos $(\operatorname{Pr} \mathrm{F} \leq 0.01)$.

\section{Conclusiones}

La dinámica de la acumulación de biomasa aérea fue diferente entre variedades, siendo Jacona Zamorana y Festival las que acumularon mayor biomasa. Los modelos obtenidos mediante regresión lineal múltiple permitieron describir la dinámica de la concentración y extracción nutrimental de N, P, K, Ca, Mg y S en las variedades de fresa Albión, Festival, Jacona y Zamorana. Las concentraciones de nutrimentos se presentaron en el siguiente orden $\mathrm{K}>\mathrm{N}>\mathrm{Ca}>\mathrm{Mg}>\mathrm{P}>\mathrm{S}$.

Los modelos para concentración de nutrimentos pueden ser utilizados como un referente en el diagnóstico nutrimental foliar para el cultivo de fresa considerando la variedad. Similarmente, los modelos de extracción nutrimental pueden utilizarse como una herramienta de apoyo en la toma de decisiones de la fertilización en sistemas semihidropónicos o hidropónicos del cultivo de fresa.

\section{Agradecimientos}

Los autores(a) expresan su agradecimiento al Consejo Nacional de Ciencia y Tecnología (CONACYT) por su apoyo durante la realización de la investigación.

\section{Literatura citada}

Agulheiro, S. A. C. 2009. Quality of strawberry 'Camarosa' with different levels of nitrogen fertilization. Acta Hortic. 842(2):907-910.

Alcántar, G. G. y Sandoval, M. 1999. Manual de análisis químico de tejido vegetal. Publicación especial Núm. 10. Chapingo, Mexico: Sociedad Mexicana de la Ciencia del Suelo. Chapingo. Edo. de México, México. 156 p. 
Avitia, G. E. J.; Pineda, P. A. M.; Castillo, G. L. I.; Trejo, T. T.; Corona, T. E. y Cervantes, U. 2014. Extracción nutrimental en fresa (Fragaria x ananassa Duch.). Rev. Mex. Cienc. Agríc. 5(3):519-524.

Cárdenas, N. R., L.; López, P. P.; Lobit, R.; Ruiz, C. y Castellanos, M. V. C. 2006. Effects of nitrogen source on growth and development of strawberry plants. J. Plant Nutr. 29(9):1699-1707.

Castro, G. A.; Rodríguez, D. and Hoyos, M. 2005. Marginal mineral nutrition of strawberry (Fragaria $\times$ ananassa) plants grown hydroponically. Acta Hortic. 697(1):321-327.

Costa, A. F.; Teodoro, P. E.; Bhering, L. L.; Fornazier, M. J.; Andrade, J. S.; Martins, D. S. and Zanuncio, J. S. 2017. Selection of strawberry cultivars with tolerance to Tetranychus urticae (Acari: Tetranychidae) and high yield under different managements. Genetics Mol. Res. 16(2):1-9.

Daugaard, H. 2001. Nutritional status of strawberry cultivars in organic production. J. Plant Nutr. 24(9):1337-1346.

Enz, M. y Dachler C. 1998. Compendio para la identificación de los estadios fenológicos de especies mono- y dicotiledóneas cultivadas escala BBCH extendida. BBA. Limburgerhof, Alemania. $123 \mathrm{p}$.

Esteca, F. D. C. N.; Dainese C. D. N.; Rodrigues L. R.; Lourenção, A. L.; Passos, F. A. and de Moraes, G. J. 2017. Resistance of new strawberry genotypes to the two-spotted spider mite (Acari: Tetranychidae). Journal of Agricultural Science. 9(8):119-129.

Favela, C. E.; Preciado, R. P. y Benavides, M. A. 2006. Manual para la preparación de soluciones nutritivas. Cultivos sin suelo. Primera (Ed.). Torreón, Coahuila. Universidad Autónoma Agraria Antonio Narro. 53-77 pp.

Hernández, J. J. M.; Castillo, B. G. A.; Navarro, A. L. A.; Torres T. L. J.; Sahagún, C. J. y Colinas, L. M. T. 2006. Propuesta para la formulación de soluciones nutritivas en estudios de nutricion vegetal. Interciencia. 31(4):246-253.

Jamali, B.; Eshghi, S. and Taffazoli, E. 2013. Vegetative growth, yield, fruit quality and fruit and leaf composition of strawberry $c v$ 'Pajaro' as influenced by salicylic acid and nickel sprays. J. Plant Nutr. 36(7):1043-1055.

Lundy, M. 2007. Análisis del sistema producto fresa en el valle de Zamora, Michoacán, México. In: la fresa en Michoacán. Los retos del mercado. J. A. Berdegué y X. Sanclemente (eds.). $1^{\text {ra }}$ ed. Consejo Estatal de la Fresa (COEFREM AC.)-SEDAGRO. Michoacán, México. 51-64 pp.

Martínez, B. M.; Nieto, A. D.; Téliz, O. D.; Rodríguez, A. J.; Martínez, D. H.; Vaquera, H. M. T. y Carrillo, M. O. 2008. Comparación cualitativa de fresas (Fragaria x ananassa Duch.) de cultivares mexicanos y estadounidenses. Rev. Chapingo Ser. Hortic. 14(2):113-119.

Mattar, M. y Pizarro C. 2007. Determinación de la curva de absorción de nutrientes, mediante sondas extractómetras y análisis foliares en palto (Persea americana Mill) cv Hass. In: memoria VI World Avocado Congress. 12 al 16 de noviembre de 2007. Viña del Mar, Chile. 1-10 pp.

Meier, U.; Bleiholder, H.; Buhr, L.; Feller, C.; Hack, H.; Heß, M. and Zwerger, P. 2009. The BBCH system to coding the phenological growth stages of plants-history and publications. J. für Kulturpflanzen. 61(2):41-52.

Molina, E.; Salas, R. y Castro A. 1993. Curva de crecimiento y absorción de nutrimentos en fresa (Fragaria x ananasa cv. Chandler) en Alajuela. Agron. Costarric. 17(1):67-73.

Montoya, G. C. O.; Volke, H. V. H.; Trinidad, S. A. y Villanueva, V. C. 2018. Concentración nutrimental de la verdolaga (Portulaca oleracea L.) en respuesta a la fertilización con NPK. Agrociencia. 52(2):241-254. 
Muramoto, J. 2003. Nutrient analysis of organic strawberries : effect of cultivars and mycorrhizal inoculation. Organic Farming Research Foundation. California, USA. 22 p.

Nestby, R.; Lieten, F.; Pivot, D.; Raynal, L. C.; Tagliavini, M. and Evenhuis, B. 2004. Influence of mineral nutrients on strawberry fruit quality and their accumulation in plant organs. A review. Acta Hortic. 649(1):201-206.

Olivia, T.; Martínez, M.; José, L. I. C. y Calzada, E. 2017. Fresa, deficiencias y síntomas nutricionales 'una guía visual para fertilizar'. Instituto Nacional de Investigaciones Forestales, Agrícolas y Pecuarias (INIFAP). Folleto técnico núm. 36.34 p.

Orona, C. I.; Cueto, W. J. A.; Murillo, A. B.; Santamaria, C. J.; Flores, H. A.; Valdez, C. R. D. and Troyo, D. E. 2004. Mineral extraction of green prickly pear cactus under drip irrigation. J. Professional Association for Cactus Development. 6(1):90-101.

Petrovic, A. and Yoshid, Y. 2013. Changes in foliar ammonium concentrations in substrate-grown strawberry. J. Plant Nutr. 36(13):2099-2109.

Shaw, D. V. and Larson, K. D. 2005. United states plant patent application US 2005/0172374 P1. United States. https://patentimages.storage.googleapis.com/64/96/78/c747565d57cf97/ US20050172374P1.pdf.

SIAP. 2017. Servicio de Informacion Agroalimentaria y Pesquera. https://www.gob.mx/siap.

Steiner, A. A. 1984. The universal nutrient solution. In: Proceedings of the $6^{\text {th }}$ International Congress on Soilless Culture International. 29 de abril al 05 de mayo. Wageningen, The Netherlands. 633-650 pp.

Stewart, W. M. 2007. Consideraciones en uso eficiente de nutrientes. International Plant Nutrition Institute-IPNI. Informacion Agronómicas. 1(67):1-16.

Tagliavini, M.; Baldi, E.; Lucchi, P.; Antonelli, M.; Sorrenti, G.; Baruzzi, G. and Faedi, W. 2005. Dynamics of nutrients uptake by strawberry plants (Fragaria x ananassa Dutch.) grown in soil and soilless culture. Eur. J. Agron. 23(1):15-25.

Tagliavini, M.; Baldi, E.; Nestby, R.; Raynal, L. C.; Lieten, P.; Salo, T. and Faedi, W. 2004. Uptake and partitioning of major nutrients by strawberry plants. Acta Hortic. 649(1):197-200.

Vega, D. R. 2007. Historia de la Introducción del cultivo de la fresa al valle de Zamora, Michoacán (1938-2006). $2^{\text {da }}$ edición. Fundación Produce Michoacán. Michoacán, México. 30-70 pp.

Volke, H. V. 2008. Estimación de funciones de respuesta para información de tipo no experimental, mediante regresión. Colegio de Postgraduados en Ciencias Agrícolas. Montecillo, Estado de México, México. 113 p.

Wójcik, P. and Lewandowski, M. 2003. Effect of calcium and boron sprays on yield and quality of 'Elsanta' strawberry. J. Plant Nutr. 26(3):671-682.

Woldb, J. S. and Hutchisona, W. D. 2003. Comparison of economic and plant phenology-based thresholds for management of Lygus lineolaris (Hemiptera: Miridae) in Minnesota strawberries. J. Econ. Entomol. 96(5):1500-1509.

Yadav, S. K.; Khokhar, U. U.; Sharma, S. D. and Kumar, P. 2016. Response of strawberry to organic versus inorganic fertilizers. J. Plant Nutr. 39(2):194-203. 